\title{
Tuberculous uveitis: association between anti-tuberculous therapy and clinical response in a non-endemic country
}

\author{
Kristina L. Bajema', Kaivon Pakzad-Vaezi ${ }^{2}$, Thomas Hawn ${ }^{1}$ and Kathryn L. Pepple ${ }^{2^{*}}$ (D)
}

\begin{abstract}
Background: The study aims to report the association between successful uveitis control following antituberculous therapy (ATT) for uveitis associated with a positive tuberculosis (TB) screening test in a low endemic setting. A retrospective chart review of cases between 2010 and 2017 at a tertiary uveitis referral center in the United States of America was conducted. Subjects with any form of uveitis, a positive TB interferon-gamma release assay or tuberculin skin test, and negative evaluation for other causes of uveitis were included. ATT was recommended in all cases and completed therapy was categorized as either adequate or inadequate for active TB infection. Location and severity of inflammation and the use of local versus systemic corticosteroid therapy was assessed at presentation and again after recommendation of ATT.

Results: Thirty-one eyes of 20 individuals were identified. Uveitis activity improved in 22 eyes of 15 patients (13 treated adequately for active TB, 2 not adequately treated). Nine eyes of 5 patients did not have improved activity ( 1 adequately treated, 4 not adequately treated). All 9 individuals presenting with posterior or panuveitis who improved were adequately treated whereas the remaining 2 who did not improve were not $(P 0.02)$. Among those with anterior or intermediate uveitis, no clear treatment patterns were observed between those who did and did not improve ( $P$ 0.50). Six individuals (30\%) experienced significant ATT-related adverse effects.
\end{abstract}

Conclusions: In a non-endemic setting, ATT for uveitis associated with a positive TB screening test may provide benefit in controlling ocular inflammation, particularly for those with posterior or panuveitis. The role for ATT in anterior or intermediate uveitis is less clear.

Keywords: Tuberculous uveitis, Ocular tuberculosis, Mycobacterium tuberculosis, Anti-tuberculous therapy

\section{Background}

In 2014, 9.6 million people worldwide developed TB leading to 1.5 million deaths [1]. In the United States of America (USA), 9421 cases were reported during this period with $21 \%$ manifesting as extrapulmonary disease [2]. Ocular findings occur in $1-2 \%$ of patients with active TB.

Diagnosis of tuberculous uveitis (TBU) depends largely on history and characteristic exam findings [3]. Though ocular fluid can be sampled for acid-fast smear, culture, or M. tuberculosis polymerase chain reaction (MTB-PCR), this

\footnotetext{
* Correspondence: kpepple@uw.edu

${ }^{2}$ Department of Ophthalmology, University of Washington, 325 9th Avenue, Box 359608, Seattle, WA 98104, USA

Full list of author information is available at the end of the article
}

is often not performed due to low test sensitivity and morbidity related to an invasive procedure. Screening for past exposure to TB can also be done by an interferon-gamma release assay (IGRA) such as QuantiFERON-TB Gold (QFT-G). However, this cannot distinguish between latent and active infection, and while specificity is generally high in non-BCG-vaccinated individuals [4], less is known about test characteristics in the setting of TBU [5-7].

Successful outcomes in patients treated with ATT, when measured by improvement in inflammation or non-recurrence of inflammation, range from 47 to $89 \%$ [8]. This variability is due in part to heterogeneity in treatment practices as well as in clinical presentation. Though studies have suggested benefit of ATT for TBU, less is known about the role of therapy in different 
disease manifestations. To better understand the association between successful uveitis control in patients with presumed TBU and ATT in a low endemic setting, we report outcomes of 20 patients with a presumptive diagnosis seen at an academic referral center in the USA.

\section{Methods}

We conducted a retrospective review of medical records of patients seen at the ophthalmology clinic at Harborview Medical Center at the University of Washington between 2010 and 2017. Individuals were included if ophthalmology assessment raised a concern for TBU. Specifically, criteria included individuals with clinical signs of uveitis; evidence of tuberculous infection by either tuberculin skin test (TST), QFT-G, or ocular sampling for MTB-PCR; and exclusion of non-tuberculous infectious and non-infectious etiologies by screening tests. Non-tuberculous etiologies included syphilis (syphilis IgG or T. pallidum particle agglutination assay (TPPA)), rheumatoid arthritis (rheumatoid factor (RF)), lupus (antinuclear antibodies (ANA)), sarcoidosis (angiotensin-converting enzyme (ACE) and chest radiography), and toxoplasmosis (toxoplasma IgM and IgG). All patients underwent independent clinical evaluation by an infectious disease specialist following referral from ophthalmology prior to initiation of ATT.

Data were abstracted from the De-identified Clinical Data Repository (DCDR) which contains information collected from the University of Washington clinical systems between 2010 and 2017. A Microsoft Amalga query was run on the DCDR database using the keyword search term "TB of the eye." No additional cases of interest were captured by further searches which included tuberculous uveitis, tuberculous chorioretinitis, tuberculous conjunctivitis, tuberculous episcleritis, tuberculous interstitial keratitis, tuberculous iridocyclitis, and tuberculous keratitis. Several additional individuals receiving ongoing clinical care were also included in the study.

Abstraction of demographics (age, gender, country of origin), TB risk factors, prior active or latent TB infection (LTBI), medical co-morbidities, time from symptom onset to diagnosis, and site of involvement was performed using a standardized data tool. Site of inflammation was categorized by a fellowship-trained uveitis specialist as anterior uveitis, intermediate uveitis, posterior uveitis, or panuveitis according to Standardization of Uveitis Nomenclature (SUN) guidelines [9]. Unilateral or bilateral disease was also ascertained. Information collected on diagnostic evaluation included TST, QFT-G, ocular sampling for MTBPCR, sputum sampling for acid-fast bacilli (AFB) smear and culture, chest radiograph or computed tomography (CT), and human immunodeficiency virus (HIV) test.

Information on recommended treatment was also documented and included anti-tuberculous drugs, systemic steroids, topical steroids, and any drug-related toxicities.
Completed therapy was categorized by infectious diseasetrained clinicians according to the following scheme: (1) Adequate active ATT (adequately treated for active TB): defined according to standard treatment for drugsusceptible pulmonary TB [10] and included individuals in whom a fluoroquinolone was substituted for ethambutol during the intensive phase. (2) Adequate alternative active ATT: this included patients who were either not started on a four-drug regimen or completed less than 2 months of intensive phase therapy but received at least 6 months of daily isoniazid- or rifampin-containing combined therapy. These treatment courses are sufficient for different types of paucibacillary disease such as culture negative pulmonary $\mathrm{TB}$ which is often treated with a shortened 4-month regimen per American Thoracic Society guidelines [10]. (3) Inadequate or no active ATT (inadequately treated for active TB). Inadequate active ATT did not meet any of the definitions above and included treatment for LTBI.

Finally, inflammation was reassessed after recommendation of ATT and improvement was determined using SUN criteria [9]. Improvement was defined for patients with anterior uveitis as decrease of anterior chamber (AC) cell by 2 grades or a final score of $<0.5+$ with topical corticosteroid eye drops administered no more than two times daily (low-dose topical therapy). For patients with anterior and intermediate uveitis, a decrease in vitreous haze by two grades or a final score of $<0.5+$ with low dose topical corticosteroid therapy or low-dose oral corticosteroid ( $<10 \mathrm{mg}$ daily) was considered improvement. For patients with posterior uveitis maintenance of quiescence of any active choroidal, retinal, or vascular lesions noted at presentation with low-dose oral corticosteroid ( $<10 \mathrm{mg}$ daily) therapy after an initial pulse and taper was considered improvement. Patients that required topical corticosteroid therapy $>2$ drops daily, or corticosteroid sparing therapy with or without oral corticosteroids were designated not improved. Improvement was ascertained within a 1-month window of the date 6 months after cessation of ATT when possible. In the absence of long-term follow-up, the clinical exam at the last recorded follow-up was used. If patients declined ATT therapy, the study endpoint was determined at the office visit falling between 6 and 12 months after initiation of anti-inflammatory therapy. If improvement in inflammation was observed before initiation of ATT, treatment was reclassified as no ATT group for final analysis.

We used Fisher's exact test to compare clinical improvement according to primary predictors of interest, adequate ATT and site of inflammation. To examine possible confounding effects, we used Fisher's exact test to compare associations between clinical improvement and age (stratified as $<60$ and $\geq 60$ years), sex, birth in a high-burden country, duration of symptoms (stratified as 
$\leq 1$ and $>1$ year), and underlying immunosuppression. The study was reviewed and approved by the University of Washington Institutional Review Board.

\section{Results}

Thirty-one eyes of 20 patients with TBU were identified. Full details regarding baseline demographic and clinical characteristics are presented in Table 1. Of note, 16 patients were born outside the USA with 7 being from high TB-burden countries [11]. Five patients had underlying immunosuppressing conditions: 1 with psoriasis receiving methotrexate; 2 with chronic hepatitis $C$; 1 with end-stage renal disease status post renal transplant on mycophenolate, prednisone, and tacrolimus; and 1 with diabetes.

Eleven patients presented with bilateral disease while 9 had unilateral findings. Six presented with anterior uveitis, 3 had anterior and intermediate uveitis, 3 presented with posterior uveitis, and 8 had panuveitis. Alternative etiologies for uveitis were excluded as described in "Methods".

ATT was initiated in 17 of 20 individuals (Table 2). Fourteen received four drugs for at least part of their therapy. Median duration among those completing therapy was 6 months (IQR range 6-8 months). All patients were treated with topical, local, or systemic corticosteroids to achieve inflammation quiescence per consensus treatment guideline for patients with uveitis [9]. Twelve individuals required treatment with systemic steroids for initial inflammation control. After inflammation control was achieved, corticosteroid tapering was initiated at the treating physician's discretion but typically proceed by $10 \mathrm{mg}$

Table 1 Demographic and clinical characteristics of patients diagnosed with tuberculous uveitis

\begin{tabular}{ll}
\hline Baseline characteristics & \\
\hline Median age (IQR), years & $38(31-58)$ \\
Men, $n / N(\%)$ & $12 / 20(60 \%)$ \\
Foreign born, $n / N(\%)$ & $16 / 18(89 \%)$ \\
$\quad$ Born in high TB-burden country & $7 / 18(39 \%)$ \\
Prior clinical history of active TB, $n / N(\%)$ & $3 / 20(15 \%)$ \\
Prior clinical history of LTBI, $n / N(\%)$ & $4 / 20(20 \%)$ \\
Median time from onset of symptoms to & $24(3-96)$ \\
ATT (IQR), months & \\
Positive screening test for LTBI & $20 / 20(100 \%)$ \\
$\quad$ QFT-G & 18 \\
$\quad$ TST & 2 \\
Abnormal chest imaging, $n / N(\%)^{\mathrm{a}}$ & $2 / 19(11 \%)$ \\
HIV test negative, $n / N$ tested (\%) & $14 / 14(100 \%)$ \\
Active concurrent TB, $n / N(\%)$ & $0 / 20(0 \%)$ \\
\hline
\end{tabular}

${ }^{a}$ Active pulmonary TB excluded in both cases with negative sputum tests for AFB smear, mycobacterial culture, and MTB-PCR per day per week for daily systemic doses $>20 \mathrm{mg} /$ day and by $2.5-5 \mathrm{mg} /$ day/week for doses between 5 and $20 \mathrm{mg} /$ day. Topical therapy was tapered by one drop/day/ week once AC cell was improved to $1+$ cell or less, and as long as quiescence was maintained.

At the study endpoint, 22 eyes of 15 patients were improved-20 eyes of 13 patients who were adequately treated for active TB and 2 eyes of 2 patients who were not. In 1 of the untreated cases, inflammation improved before ATT was initiated. Nine eyes of 5 patients were not improved; of these, only 2 eyes in 1 patient were adequately treated while 7 eyes in the remaining 4 patients were not (1 patient treated for LTBI, 1 inadequately treated, and 2 not treated; $P 0.01$ comparing proportion improved in adequate versus inadequate ATT groups). All 9 individuals presenting with posterior or panuveitis who improved were adequately treated whereas the remaining 2 who did not improve were not (1 inadequately treated, 1 untreated; $P$ 0.02) [Fig. 1]. Among 9 patients with anterior or intermediate uveitis, no clear treatment patterns were observed between those who did and did not improve though 1 improved without any therapy $(P 0.50)$. There was no association between clinical improvement and age, sex, birth in a high-burden country, duration of symptoms, or underlying immunosuppression.

Adverse drug effects from ATT were common. While 4 developed only mild symptoms, 6 (30\%) had severe reactions or toxicities requiring a temporary or permanent cessation of the offending agent. These included 2 individuals with drug-induced hepatitis, 1 with severe allergic reaction, and 3 with nausea and vomiting.

\section{Discussion}

In a non-endemic setting, we found that patients with posterior or panuveitis who received adequate ATT were able to achieve ocular inflammation control more often than patients that did not have adequate treatment. In contrast, no such association was seen in patients with anterior and intermediate uveitis.

Ocular TB is thought to represent either active mycobacterial infection or a delayed-hypersensitivity reaction to bacilli present at a different site in the body. Support for the former hypothesis is based on studies of patients with ocular inflammation in which $M$. tuberculosis was identified in ocular specimens through histopathology or nucleic acid amplification [12-14]. While ATT is indicated for active infection, it is not expected to be of therapeutic benefit in treating an immune-mediated reaction. The hypothesis of active infection is supported by studies that demonstrate better clinical response in the setting of longer ATT duration. Compared to no treatment, ATT for more than 9 months was associated with a significant reduction in recurrence of inflammation [15], an effect than was not significant in shorter 
Table 2 Clinical presentation, treatment details, and clinical outcomes of 20 study patients

\begin{tabular}{|c|c|c|c|c|c|c|c|}
\hline Case & Sex & Uveitis presentation & $\begin{array}{l}\text { Unilateral or } \\
\text { bilateral }\end{array}$ & $\begin{array}{l}\text { Treatment regimen } \\
\text { started }\end{array}$ & $\begin{array}{l}\text { Duration therapy } \\
\text { completed (months) }\end{array}$ & $\begin{array}{l}\text { Additional } \\
\text { systemic steroids }\end{array}$ & $\begin{array}{l}\text { Uveitis } \\
\text { improved }\end{array}$ \\
\hline 1 & M & Panuveitis ${ }^{a, b}$ & Bilateral & $\mathrm{RH}^{\mathrm{d}}$ & 6 & No & Yes \\
\hline 2 & M & Posterior uveitis & Unilateral & $\mathrm{RHZM}$ & 8 & No & Yes \\
\hline 3 & $\mathrm{~F}$ & Panuveitis & Unilateral & $\mathrm{RHZ}$ & 6 & No & Yes \\
\hline 4 & M & Anterior uveitis & Unilateral & RHZE & 6 & No & Yes $^{\mathrm{e}}$ \\
\hline 5 & $\mathrm{~F}$ & Panuveitis $^{a}$ & Unilateral & RHZE & 6 & Yes & Yes \\
\hline 6 & M & Anterior uveitis & Unilateral & - & - & No & Yes \\
\hline 7 & $\mathrm{~F}$ & Anterior uveitis & Bilateral & $\mathrm{RHZE}$ & 18 & Yes & No \\
\hline 8 & M & Posterior uveitis ${ }^{\mathrm{b}, \mathrm{c}}$ & Bilateral & - & - & Yes & No \\
\hline 9 & M & Panuveitis & Unilateral & $\mathrm{RHZE}$ & 9 & Yes & Yes \\
\hline 10 & M & Anterior uveitis ${ }^{c}$ & Bilateral & $\mathrm{RHZE}$ & 6 & Yes & Yes \\
\hline 11 & $\mathrm{~F}$ & Panuveitis ${ }^{a, b}$ & Bilateral & RHZE & 6 & Yes & Yes \\
\hline 12 & M & Posterior uveitis ${ }^{\mathrm{b}}$ & Unilateral & $\mathrm{RHZM}$ & 5 & Yes & Yes \\
\hline 13 & $\mathrm{~F}$ & $\begin{array}{l}\text { Anterior, intermediate } \\
\text { uveitis }^{\mathrm{a}}\end{array}$ & Bilateral & RHZM & 9 & No & Yes \\
\hline 14 & $\mathrm{~F}$ & $\begin{array}{l}\text { Anterior, intermediate } \\
\text { uveitis }^{\mathrm{a}}\end{array}$ & Bilateral & $\mathrm{RHZM}$ & 6 & No & Yes \\
\hline 15 & F & Panuveitis ${ }^{a, c}$ & Bilateral & RHZE & Sporadic & Yes & No \\
\hline 16 & M & $\begin{array}{l}\text { Anterior, intermediate } \\
\text { uveitis }^{b}\end{array}$ & Bilateral & - & - & Yes & No \\
\hline 17 & M & Anterior uveitis ${ }^{\mathrm{a}}$ & Unilateral & RHZE & 7 & No & Yes \\
\hline 18 & $\mathrm{~F}$ & Anterior uveitis & Unilateral & RPTH & 3 & Yes & No \\
\hline 19 & M & Panuveitis uveitis & Bilateral & RHZE & 6 & Yes & Yes \\
\hline 20 & M & Panuveitis & Bilateral & RHZL & 7 & Yes & Yes \\
\hline
\end{tabular}

${ }^{\mathrm{a}}$ Cases born in a high TB-burden country: 1, 5, 11, 13, 14, 15, 17

${ }^{b}$ Cases with underlying immunosuppression: 1, 8, 11, 12, 16

${ }^{\mathrm{C} C}$ Cases with reported prior active TB: 8, 10, 15

${ }^{\mathrm{d}}$ ATT abbreviations: R-rifampin, RPT—rifapentine, H-isoniazid, Z-pyrazinamide, E-ethambutol, M-moxifloxacin

enflammation improved before the start of ATT

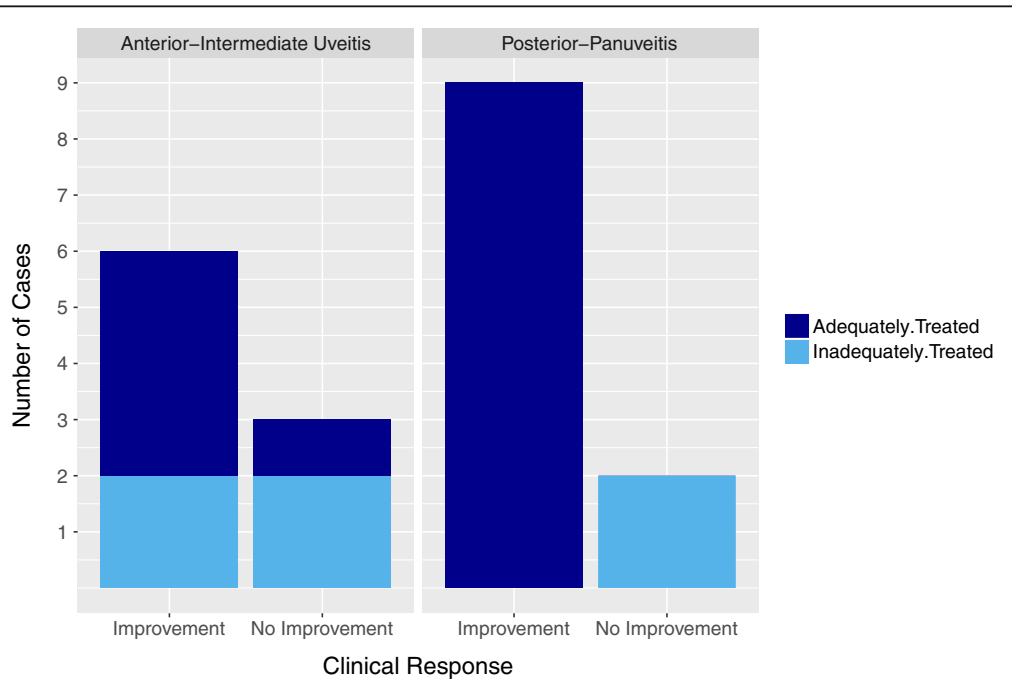

Fig. 1 Clinical improvement stratified by initial uveitis presentation and adequate or inadequate ATT for active TB. Numbers presented as individuals 
treatment durations. In another study, the risk of inflammation recurrence was significantly reduced among those receiving more than 9 months of ATT compared with shorter course therapy [16]. Other studies of treatment response are more equivocal with regard to the hypothesis of active infection. In a meta-analysis by Kee et al., the pooled relative risk of ATT for improvement in inflammation related to TBU was only 1.17 (95\% CI $0.74-1.85$ ) and 1.42 (95\% CI 1.24-1.63) for non-recurrence of inflammation. Thus the role for ATT in TBU remains unclear. Our study suggests that multi-drug therapy for active TB provides higher cure rates than interrupted regimens or no treatment. Taken together, our data support a model of active infection as opposed to a pure hypersensitivity response to M. tuberculosis antigens in the absence of live bacilli.

In our cohort, individuals with posterior or panuveitis were more responsive to ATT than those with anterior or intermediate uveitis. Few studies have examined treatment outcomes in the context of differing sites of ocular inflammation. Ang et al. showed a trend toward recurrence of inflammation following ATT comparing intermediate to anterior uveitis as well as non-significant reduction in recurrence of inflammation comparing posterior to anterior uveitis [15]. In contrast, Patel et al. described an increased risk of relapse comparing posterior to other forms of uveitis including panuveitis [17]. However, the authors noted that relapse occurred less often among those receiving a full ATT course and that relapsed cases responded to extended therapy. In a large retrospective study of 360 patients with uveitis and a positive TB screening test, Bansal et al. also reported an overall significant reduction in recurrence of inflammation when ATT was added to corticosteroid therapy compared to corticosteroids alone [18]. Though anatomic location of uveitis was not found to be a significant predictor of recurrence risk, their data is consistent with the trend identified here; patients with anterior or intermediate uveitis are more likely to have continued disease activity despite combined treatment with ATT and corticosteroids. Although different treatment approaches are not routinely employed based on the location of ocular inflammation, future studies should address this possibility. In particular, anterior and intermediate uveitis may require different treatment than other forms of uveitis.

Adverse drugs effects related to ATT were common; one third of treated patients experienced severe toxicities that required either temporary or permanent drug discontinuation. This is much higher than what has been reported in previous studies [8] and may reflect differences in age, co-morbidities, and toxicity monitoring.

Our study is limited by small numbers, heterogeneity in ATT and corticosteroid regimens, and need for longterm follow-up to evaluate recurrence of inflammation. Though ATT was classified dichotomously as adequate or inadequate for active TB, LTBI or partial treatment courses can be expected to have some benefit in paucibacillary infection. As only two patients were treated in this manner, the impact on interpretation of treatmentrelated outcomes was minimal. Incidental lack of ATT in some cases did afford the opportunity to observe treatment-independent responses which has not been extensively reported in the literature. Altogether, these findings suggest a role for ATT among individuals with posterior uveitis and panuveitis. Lack of clear benefit in anterior and intermediate uveitis combined with high risk of adverse events may warrant a period of observation before considering ATT. Our study also lacks a control group of uveitis patients without a positive TB screening test; therefore, conclusions about the benefit of ATT on uveitis control and the mechanism underlying any benefit are limited. Ultimately, a randomized trial is needed to fully address this question.

\section{Conclusions}

In agreement with prior studies in paitents with uveitis in TB endemic retions, in a TB non-endemic setting, ATT for uveitis associated with a positive TB screening test may provide benefit in controlling ocular inflammation, particularly for those with posterior or panuveitis. The role for ATT in anterior or intermediate uveitis is less clear.

\section{Acknowledgements \\ The authors would like to thank Nic Dobbins for assistance with DCDR data abstraction. \\ Funding \\ This work was supported by the University of Washington Department of Medicine De-identified Clinical Data Repository (DCDR) Observational Research Pilot Grant. KLB is supported by the Host Defense Training in Allergy and Infectious Diseases Grant T32 Al007044. KLP is supported by NIH NE K08EY023998 and a career development award from Research to Prevent Blindness.}

\section{Authors' contributions}

$\mathrm{KLB}, \mathrm{KPV}$, and $\mathrm{KP}$ were involved in the study design, data analysis, and manuscript drafting. TH was involved in data interpretation and manuscript revision. All authors read and approved the final manuscript.

\section{Ethics approval and consent to participate}

The study was reviewed and approved by the University of Washington Institutional Review Board.

\section{Competing interests}

The authors declare that they have no competing interests.

\section{Publisher's Note}

Springer Nature remains neutral with regard to jurisdictional claims in published maps and institutional affiliations.

\section{Author details}

'Department of Medicine, Division of Allergy and Infectious Diseases, University of Washington, 1959 NE Pacific St., Box 356423, Seattle, WA 98195, USA. ${ }^{2}$ Department of Ophthalmology, University of Washington, 325 9th Avenue, Box 359608, Seattle, WA 98104, USA. 
Received: 30 August 2017 Accepted: 25 September 2017

Published online: 04 October 2017

\section{References}

1. World Health Organization. Tuberculosis. Available at: http://www.who.int/ mediacentre/factsheets/fs104/en/. Accessed March 24, 2017

2. Centers for Disease Control and Prevention (CDC). Reported tuberculosis in the United States, 2014. Atlanta: USA Department of Health and Human Services, CDC; 2015

3. Ang M, Chee SP (2017) Controversies in ocular tuberculosis. Br J Ophthalmol 101(1):6-9

4. Pai M, Zwerling A, Menzies D (2008) Systematic review: T-cell-based assays for the diagnosis of latent tuberculosis infection: an update. Ann Intern Med 149(3):177-184

5. La Distia NR, van Velthoven ME, Ten Dam-van Loon NH et al (2014) Clinical manifestations of patients with intraocular inflammation and positive QuantiFERON-TB gold in-tube test in a country nonendemic for tuberculosis. Am J Ophthalmol 157(4):754-761

6. Caspers L, Makhoul D, Ebraert H, Michel O, Willermain F (2014) Clinical manifestations of patients with intraocular inflammation and positive QuantiFERON-TB gold in-tube test in a country nonendemic for tuberculosis. Am J Ophthalmol 158(3):646-647

7. Kuznetcova TI, Sauty A, Herbort CP (2012) Uveitis with occult choroiditis due to Mycobacterium kansasii: limitations of interferon-gamma release assay (IGRA) tests (case report and mini-review on ocular non-tuberculous mycobacteria and IGRA cross-reactivity). Int Ophthalmol 32(5):499-506

8. Kee AR, Gonzalez-Lopez JJ, Al-Hity A et al (2016) Anti-tubercular therapy for intraocular tuberculosis: A systematic review and meta-analysis. Surv Ophthalmol 61(5):628-653

9. Jabs DA, Nussenblatt RB, Rosenbaum JT (2005) Standardization of uveitis nomenclature for reporting clinical data. Results of the First Internationa Workshop. Am J Ophthalmol 140(3):509-516

10. Nahid P, Dorman SE, Alipanah N et al (2016) Official American Thoracic Society/Centers for Disease Control and Prevention/Infectious Diseases Society of America Clinical Practice Guidelines: Treatment of DrugSusceptible Tuberculosis. Clin Infect Dis 63(7):e147-ee95

11. World Health Organization (WHO). Use of high burden country lists for TB by WHO in the post-2015 era. Geneva: WHO; 2015.

12. Biswas J, Madhavan HN, Gopal L, Badrinath SS (1995) Intraocular tuberculosis. Clinicopathologic study of five cases. Retina 15(6):461-468

13. Wroblewski KJ, Hidayat AA, Neafie RC, Rao NA, Zapor M (2011) Ocular tuberculosis: a clinicopathologic and molecular study. Ophthalmology 118(4):772-777

14. Gupta V, Gupta A, Rao NA (2007) Intraocular tuberculosis-an update. Surv Ophthalmol 52(6):561-587

15. Ang M, Hedayatfar A, Wong W, Chee SP (2012) Duration of anti-tubercula therapy in uveitis associated with latent tuberculosis: a case-control study. Br J Ophthalmol 96(3):332-336

16. Agrawal R, Gupta B, Gonzalez-Lopez JJ et al (2015) The role of antitubercular therapy in patients with presumed ocular tuberculosis. Ocul Immunol Inflamm 23(1):40-46

17. Patel SS, Saraiya NV, Tessler HH, Goldstein DA (2013) Mycobacterial ocular inflammation: delay in diagnosis and other factors impacting morbidity. JAMA Ophthalmol 131(6):752-758

18. Bansal R, Gupta A, Gupta V, Dogra MR, Bambery P, Arora SK (2008) Role of anti-tubercular therapy in uveitis with latent/manifest tuberculosis. Am J Ophthalmol 146(5):772-779

\section{Submit your manuscript to a SpringerOpen ${ }^{\circ}$ journal and benefit from:}

- Convenient online submission

- Rigorous peer review

- Open access: articles freely available online

- High visibility within the field

- Retaining the copyright to your article 